\title{
Immediate Complete Overdenture for Esthetic Consideration of the Upper Edentulous Ridge - A Case Report
}

\author{
Olivia Budihargono, Nike Hendrijantini and Rostiny \\ Department of Prosthodontics, Faculty of Dental Medicine, Universitas Airlangga, Jalan Mayjend Prof. Dr. Moestopo No. \\ 47, Surabaya-Indonesia. \\ nike-h@fkg.unair.ac.id
}

Keywords: Esthetics, Immediate Denture, Overdenture.

\begin{abstract}
Background: Nowadays esthetics is one of the main considerations. So, dentures not only rehabilitate functions, but esthetics as well. The problem is that "conventional denture making" requires several steps which sometimes force a patient into a toothless phase which affects both functional and esthetical aspects. In some cases, the patients are unable to endure this condition due to esthetic reasons. Immediate dentures will be the best solution for them. Purpose: To provide information about immediate complete overdenture design and fabrication. Case: A 61-year-old female patient, an active physician, came to the Dental Hospital of Airlangga University, complaining about her upper partial denture and lower complete denture, which had been worn for 10 years. They weren't comfortable to wear anymore. She wanted new dentures, without the toothless phase. The remaining teeth were 16, 13, 12, and 22. 13 and 22 were used as retainers for the upper partial denture but the inclination and vitality of the teeth were bad. Case management: Immediate complete overdenture was made for the upper edentulous ridge. Immediate treatment was chosen because of the esthetic consideration. Overdenture treatment was chosen because it preserves the bone dimensions. 16 and 12 were extracted, while 13 and 22 were endodontically treated. The complete denture was then fabricated by cutting of 13 and 22 on the functional model. After the complete denture had been fabricated, the patient's teeth 13 and 22 were decaputated as high as the gingival level and then restored with GIC on the surface. The denture was then inserted immediately after the decaputations. Discussion: An immediate denture is a denture which is made first before decaputation or extraction, then immediately inserted into the mouth after the tooth decaputation or extraction. Designing an immediate denture will give some advantages to patients, especially from the esthetic aspect. An overdenture is a good treatment for patients whose condition of teeth crowns is bad but periodontal tissue and teeth roots are in good condition. In conclusion, an immediate denture is the best treatment where esthetics is the main consideration.
\end{abstract}

\section{INTRODUCTION}

Conventional denture fabrication requires preliminary treatments which sometimes forces a patient into a toothless phase. Treatments such as extraction or decaputation of the remaining teeth before complete denture fabrication will surely force the patient into such phase. This condition will affect both functional and esthetical aspects.

In some cases, the patients are unable to undergo this toothless phase due to esthetic reasons associated with their professions or activities. An immediate denture will be the best solution for these cases. Some advantages of an immediate denture are that patients can avoid uncomfortable chewing and will not be ashamed because they will not have to go through the edentulous phase.

\section{CASE}

A 61-year-old female patient, an active physician, came to the Dental Hospital of Airlangga University, complaining about her upper right canine which is used as a retainer for her upper partial denture. That tooth caused pain although it had been treated endodontically. The second complaint was that her upper partial denture and lower complete denture, which had been worn for 10 years, weren't comfortable to wear anymore. She wanted new dentures, without the toothless phase. 
In clinical examination, we found there were 4 teeth remaining in the upper jaw. They were 16 (gangrene radix), 13, 12 (gangrene radix), and 22. 13 and 22 were used as retainers for the upper partial denture but the condition of the teeth's crowns was bad, both inclination and vitality. The diagnosis was chronic apical periodontitis et causa gangrene radix on tooth 16 and 12, chronic apical periodontitis et causa gangrene radix on tooth 13 , post endodontic treatment on tooth 22 , and flabby tissue on the anterior region of the lower jaw. From the clinical and radiographic examinations, we found that the endodontic treatments mentioned by the patient (on tooth 13 and 22) were not good. There was no guttap nor root canal sealer in tooth 13 and the treatment on tooth 22 did not reach the apex.

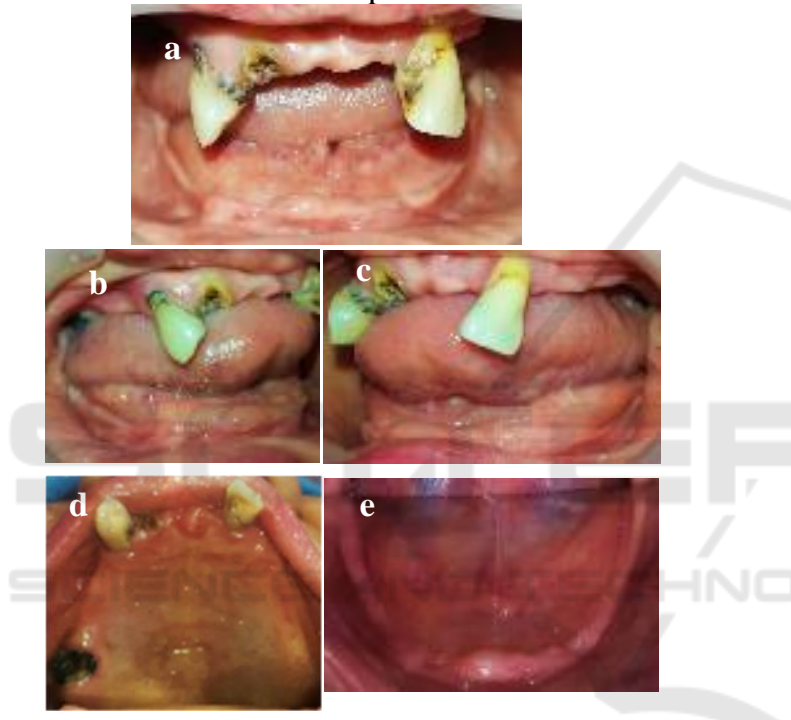

Figure 1: Intra oral condition without denture (a) anterior view, (b) lateral right view, (c) lateral left view, (d) upper occlusal view, (e) lower occlusal view.
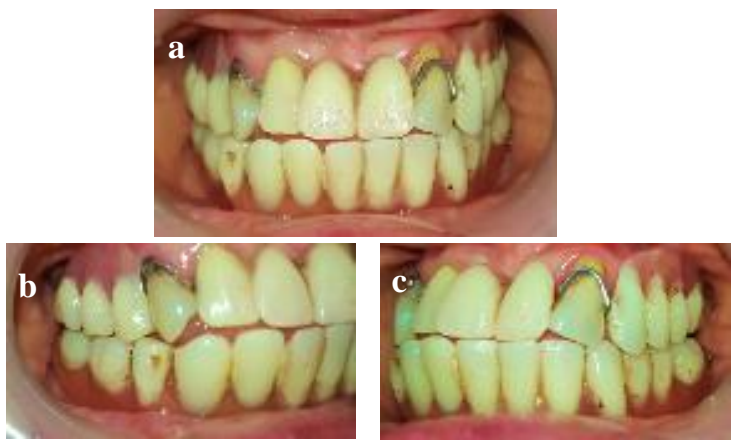

Figure 2: Intra oral condition with the old denture (a) anterior view, (b) lateral right view, (c) lateral left view.

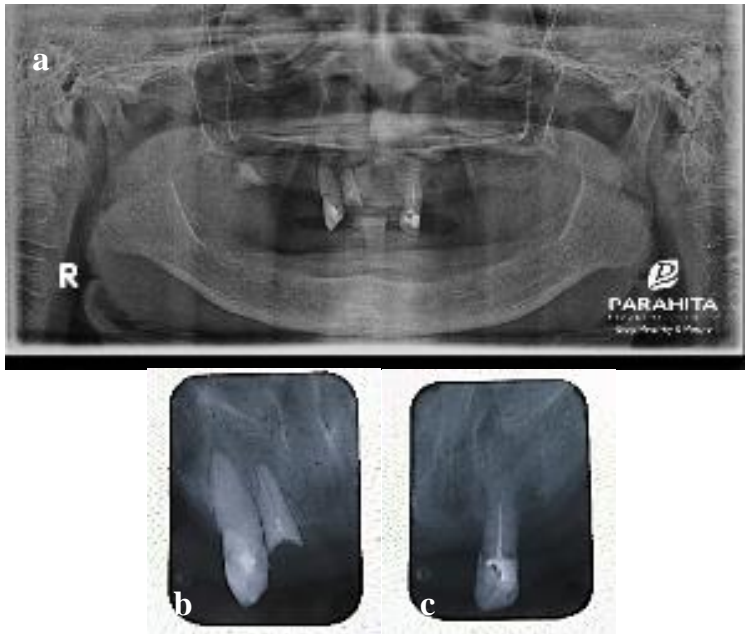

Figure 3: Radiographic examination (a) panoramic radiograph, (b) periapical radiograph showing tooth 12 and 13, (c) periapical radiograph showing tooth 22 .

\section{CASE MANAGEMENT}

Anatomical impressions were taken on the first visit. The next step was performing the preliminary treatments. Preliminary treatments were performed before the prosthodontic treatment. These treatments were extractions of 16 and 12 , and endodontic treatments for 13 and 22.

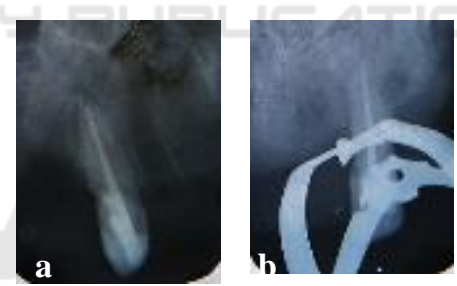

Figure 4: Periapical radiograph of tooth (a) 13 and (b) 22 after endodontic treatment.

Individual trays were made to perform border molding and to take functional impressions of the upper and lower jaw. The individual tray for the lower jaw was designed with a window in the anterior region, for taking the impression of the flabby tissue. The material used for border molding was monophase elastomer, and for taking impressions was regular body and light body elastomer. 

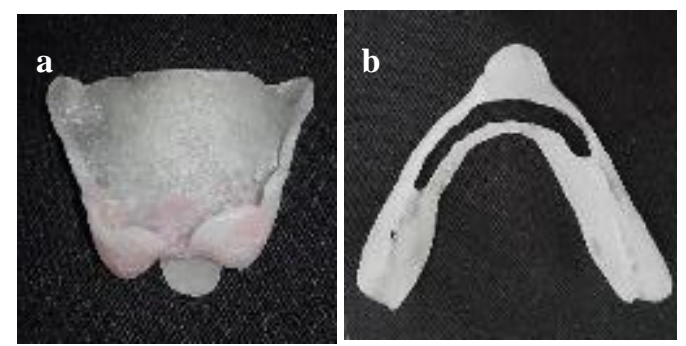

Figure 5: Individual trays for the (a) upper jaw and (b) lower jaw, with a window in the anterior region.
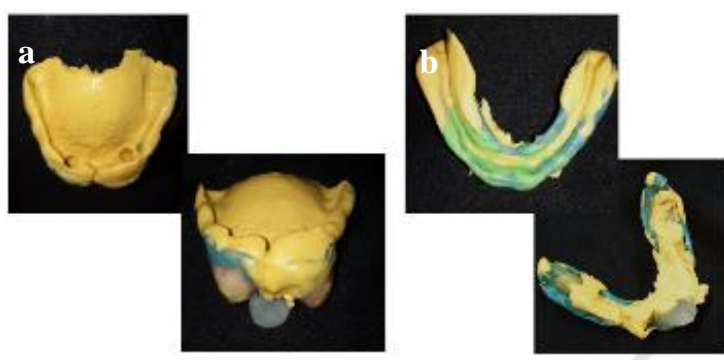

Figure 6: The impression of (a) upper and (b) lower jaw.

Dental gypsum was poured into the impression to make the functional models. Then we made upper and lower base plates from self-cured acrylic, and also a bite rim from dental wax on top of them.

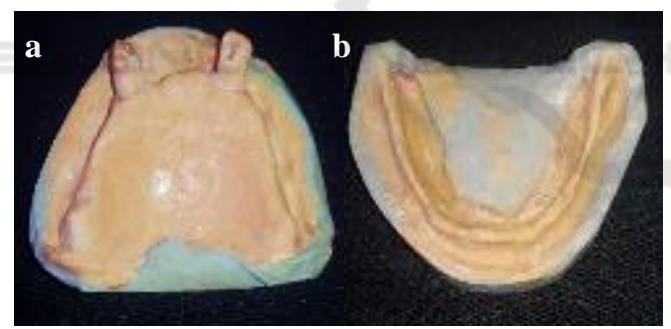

Figure 7: (a) Upper and (b) lower jaw functional models.

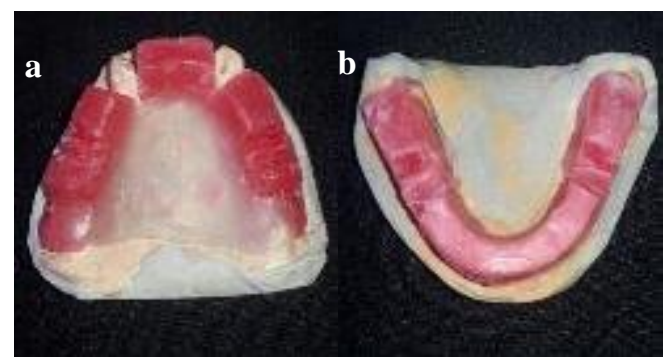

Figure 8: Base plate and bite rim on the upper and lower jaw functional models.
The base plate and bite rim were used in the next step which was bite registration. In the bite registration step, we checked the profile, vertical dimension of occlusion, midline, smile line, and canine position. We used bite registration material (Memoreg®) to transfer the correct relation of upper and lower bite rims from the patient's mouth, so we could mount the models correctly on the articulator. We also chose the artificial teeth's color together with the patient.

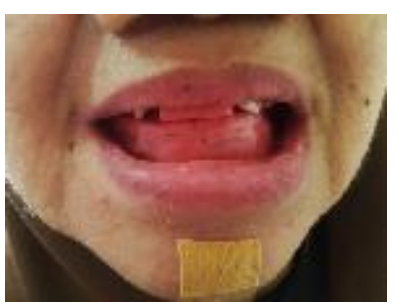

Figure 9: Bite rims inside the patient's mouth during the bite registration step.

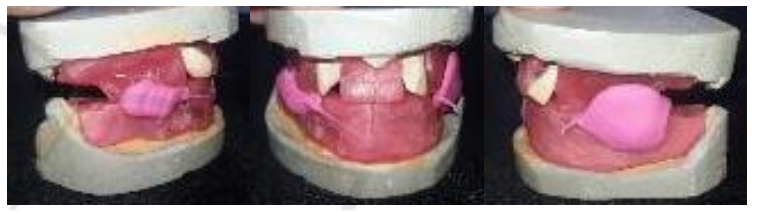

Figure 10: Upper and lower bite rims with bite registration material to transfer the correct relation of the upper and lower jaw.

The models were then mounted on a free plane articulator. After that, we set the non-immediate artificial teeth. We did the try-in of wax denture with the non-immediate artificial teeth.

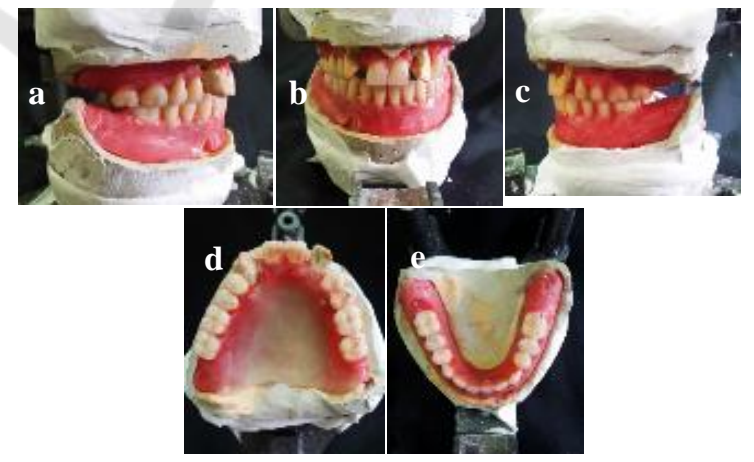

Figure 11: The setup of artificial teeth on the models mounted on a free plane articulator without artificial teeth 13, 12, 22, 23 (a) lateral right view, (b) anterior view, (c) lateral left view, (d) upper occlusal view, (e) lower occlusal view. 


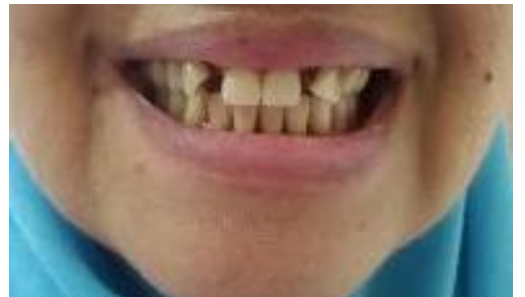

Figure 12: Wax denture try-in, without artificial teeth 13, $12,22,23$

The next step was grinding 13 and 22 on the functional model. We grinded the teeth as high as the gingival level. After the model had been grinded, we completed the artificial teeth set up by adding artificial teeth $13,12,22,23$. The wax denture was then contoured and packed to be an acrylic denture.

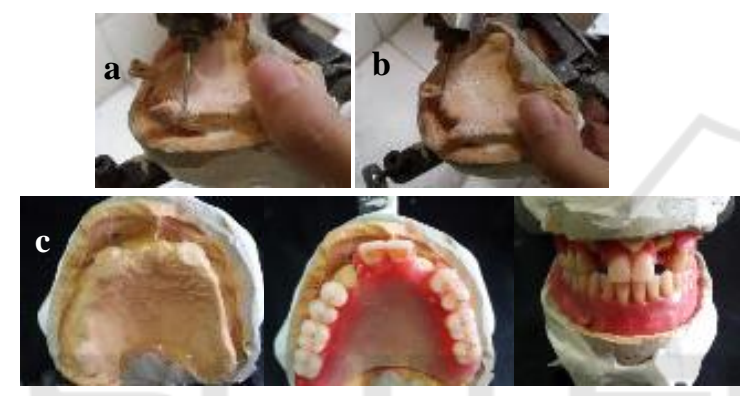

Figure 13: Grinding of teeth (a) 13 and (b) 22 on the functional model. (c) Functional model after grinding.

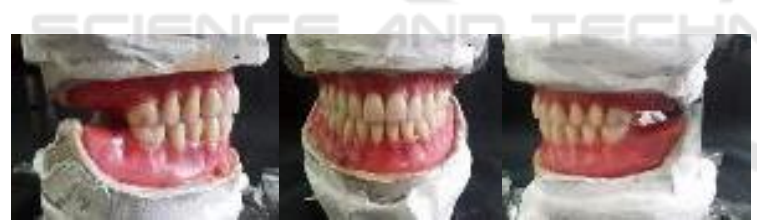

Figure 14: Completion of artificial teeth setup by adding artificial teeth $13,12,22,23$.

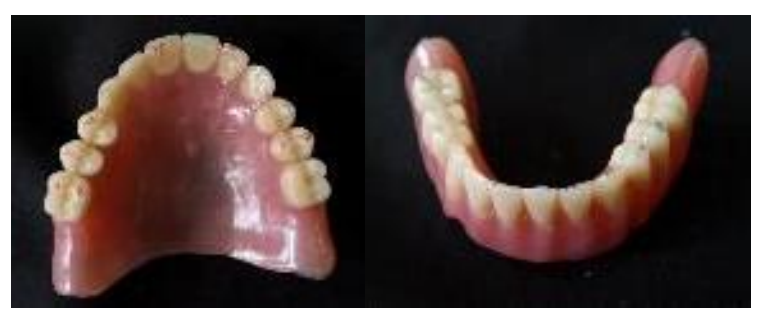

Figure 15: Acrylic denture.

On the last visit, the acrylic denture was ready to be inserted. We decaputated the patient's teeth (13 and 23). We grinded the teeth as high as the gingival level. We restored the bare-root teeth with glass ionomer cement. The denture was inserted directly after restoration of the bare-root teeth.

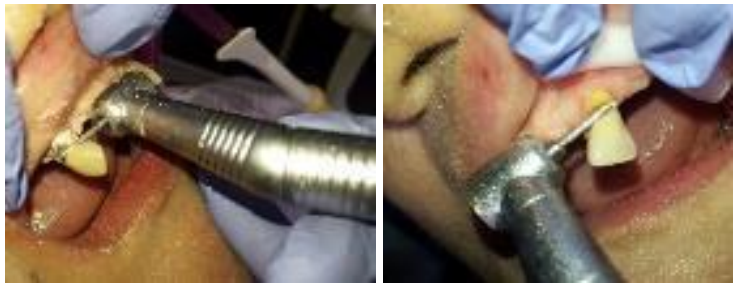

Figure 16: Decaputation of teeth 13 and 22.

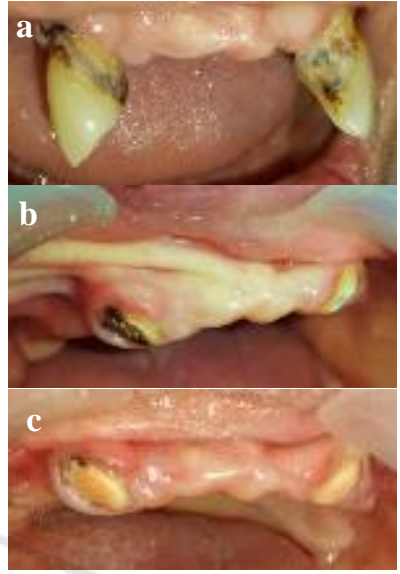

Figure 17: (a) 13 and 22 before decaputation (b) 13 and 22 after decaputation (c) 13 and 22 bare-root were restored using glass ionomer cement. 


\section{DISCUSSION}

An immediate denture is a denture which is made first before decaputation or extraction, then immediately inserted in the mouth after the tooth extraction (Bates and Stafford, 1971). Designing an immediate denture will give some advantages to patients, especially from the esthetical aspect.

In this case, the treatment choice for the upper edentulous ridge was an immediate complete overdenture. Decaputation of teeth 13 and 12 was performed right before insertion of the denture. Immediate decaputation was performed because teeth 13 and 12 functioned as the retainers of the previous partial denture, which would still be worn. If those teeth had to be decaputated first, before the fabrication of the denture, then the patient would not be able to wear her partial denture. That condition would affect both the esthetical and functional aspects, which could not be tolerated by the patient, considering her profession as an active physician who meet lots of patients every day.

An overdenture is one of treatments for patients who have teeth crowns in bad condition but periodontal tissue and teeth root in good condition, which can support the denture (Crum and Rooney, 1978). An overdenture can improve support and denture stability, increase the supporting teeth's life expectancies, and inhibit resorption of the residual ridge (Brewer and Morrow, 1980). In this case, the type of overdenture's support was bare root on teeth 13 and 22.

The patient was given instructions about how to take care of the supporting teeth (bare root 13 and 22). The patient had to maintain her oral hygiene so there will not be any food impaction on the supporting teeth. This can be achieved by brushing the teeth thoroughly and using a mouthwash. Maintaining periodontal health is one of the main factors that affect the success of overdenture treatment. The achievement of this condition depends on the effectivity of plaque control done by the patient ( $\mathrm{Ai}$ and Shiau, 2004). Topical application of fluoride by the dentist is also needed to prevent decay of the supporting teeth.

The patient was also given instructions about how to take care of the denture. The patient has to remove the denture at bedtime, because according to Watt and Gregor (1984), this is an efficient way to control caries and the progression of periodontal disease. Dentures can be cleaned using an antibacterial denture cleanser. Before being put into the denture cleanser fluid, the patient clean the denture by brushing it with gauze and soap with gentle pressure. This way of cleaning is in accordance with the opinion of Zarb (2004); it is not recommended to use toothpaste to clean a denture, because most toothpaste contains abrasive materials that can erode acrylic resin's surface. Periodic controls every 6 months have to be done to optimize this prosthodontic treatment.

\section{REFERENCES}

Ai M and Shiau YY, 2004. New Magnetic Application in Clinical Dentistry. Quintessence Publ Co. Ltd. Tokyo: p. $28-69$

Bates,J.F and Stafford GD. 1971. Immediate Complete Denture. London. The British Dental Association :11; p 18-19.

Brewer, AA, Morrow, RM 1980. Overdenture. 2nd Ed. St Louis: The C.V. Mosby Co.; p.3-11.

Crum, RJ, Rooney, GE. Jr. 1978. Alveolar Bone Loss in Overdenture: a 5-years study. J Prosthet Dent.; 40: 61013.

Watt DM, MacGregor A.R. 1984. Designing partial denture, 1st ed, John Wright \& Sons Ltd. Published under the Wright Imprint, Eidinburg. p. 90-109.

Zarb GA, Bolender CL, Eckert SE, Fenton AH, Jacob RF, 2004. Prostodontics treatment for edentulous patients, 12th, CV.Mosby Co, St. Louis. P. 123-25. 